Proyecciones Journal of Mathematics

Vol. 30, No 2, pp. 175-188, August 2011.

Universidad Católica del Norte

Antofagasta - Chile

\title{
On topological conjugacy of left invariant flows on semisimple and affine Lie groups
}

\author{
CHRISTOPH KAWAN * \\ UNIVERSITAT AUGSBURG, GERMANY \\ OSVALDO G. ROCÍO ${ }^{\dagger}$ \\ UNIVERSIDADE ESTADUAL DE MARINGÁ, BRASIL \\ ALEXANDRE J. SANTANA ‡ \\ UNIVERSIDADE ESTADUAL DE MARINGÁ, BRASIL
}

Received : February 2011. Accepted : April 2011

\begin{abstract}
In this paper, we study the flows of nonzero left invariant vector fields on Lie groups with respect to topological conjugacy. Using the fundamental domain method, we are able to show that on a simply connected nilpotent Lie group any such flows are topologically conjugate. Combining this result with the Iwasawa decomposition, we find that on a noncompact semisimple Lie group the flows of two nilpotent or abelian fields are topologically conjugate. Finally, for affine groups $G=H V, V \cong n$, we show that the conjugacy class of a left invariant vector field does not depend on its Euclidean component.
\end{abstract}

Keywords : topological conjugacy, flows, semi-simple Lie groups, nilpotent Lie group, affine groups.

Subject classification : 37B99, 54H20, 22E20

\footnotetext{
* Supported by DFG grant Co 124/17-1 within DFG priority program 1305.

${ }^{\dagger}$ Partially supported by UEM/Fundação Araucária grant $N^{\circ}$ 564/09 and 496/10

${ }^{\ddagger}$ Partially supported by UEM/Fundação Araucária grant N ${ }^{\circ} 496 / 10$
} 


\section{Introduction}

Consider two flows $\Phi$ and $\Psi$ on topological spaces $M$ and $N$. In a wider context, topological conjugacy aims to establish conditions on these flows to find a homeomorphism between $M$ and $N$ that maps $\Phi$-trajectories to $\Psi$ trajectories preserving the parametrization by time. For linear autonomous differential equations, stability theory and classification with respect to topological conjugacy are classical topics in the theory of differential equations; see, e.g., Robinson [7], Hirsch, Smale, and Devaney [4]. In the context of classification, one classical result says that in case of hyperbolicity, two linear autonomous differential equations are topologically conjugate if and only if the dimensions of the stable subspaces coincide. More specifically, take two equations $\dot{x}=A x, \dot{x}=B x$ with $A, B \in g l(d, R)$ and $x \in R^{d}$. Recall that the linear flows $\mathrm{e}^{A t} x$ and $\mathrm{e}^{B t} x$ are said to be topologically conjugate if there exists a homeomorphism $h: R^{d} \rightarrow R^{d}$ such that $h\left(\mathrm{e}^{A t} x\right)=\mathrm{e}^{B t} h(x)$ for all $x \in R^{d}$ and $t \in R$. To prove the above classical result, the existence of homeomorphic fundamental domains, one for $\mathrm{e}^{A t} x$ and another one for $\mathrm{e}^{B t} x$, is essential to construct the conjugation $h$. More generally, Ayala, Colonius, and Kliemann [1] introduced concepts of conjugacy for linear flows on control systems, the control flows, and using concepts and techniques from topological dynamics classified these linear flows. Recently, Colonius and Santana (see [2] and [3]) generalized the study of topological conjugacy to affine autonomous differential equations and to inhomogeneous bilinear control systems. They proved, in [3], that hyperbolic affine flows are topologically conjugate to their linear parts provided that an additional continuity property holds, hence the topological conjugacy between two hyperbolic affine flows depends on the topological conjugacy between their linear parts.

Recalling that $g l(d, R)$ is the Lie algebra of the Lie group $\operatorname{Gl}(d, R)$ and knowing the relation between hyperbolic elements and Iwasawa decomposition of semisimple Lie groups, it is natural ask about the above result in the case of a semisimple Lie group. Considering the semisimple Lie group $G$, the main result of this paper establishes that the flows on $G$ of two hyperbolic or nilpotent fields are topologically conjugate. The technique used in the proof consists in showing the existence of transversal sections in $G$, associated to such fields.

In Section 2, we introduce the notion of a transversal section for a flow on a topological space, which is a special kind of fundamental domain for the action (see, e.g., Katok and Hasselblatt [5] and Robinson [7]). For 
group actions, a fundamental domain is a subset of the space on which the group acts such that this subset contains exactly one point of each orbit. For a flow (an $R$-action) this is not exactly the same as our notion of transversal sections which are subsets of the state space that intersect each trajectory at exactly one time $t$, which for instance forbids fixed points. In this section, we prove that two flows on a space $X$, having homeomorphic transversal sections, are topologically conjugate. Moreover, we show that a transversal section of a flow $\Phi$ on a space $M$ lifts to a transversal section of a flow $\widetilde{\Phi}$ on $M \times P$ if the two flows are related by the canonical projection $\pi: M \times P \rightarrow M$.

In Subsection 3.1, we show that the flow of a nonzero left invariant vector field on a simply connected nilpotent Lie group always has a transversal section, and two such transversal sections are always homeomorphic. Hence, we obtain the result that two such flows are topologically conjugate. In particular, this holds for abelian Lie groups. Subsection 3.2 is devoted to the study of semisimple Lie groups. Using the Iwasawa decomposition of a semisimple Lie group and the results of Section 2 and Subsection 3.1, we are able to show that two flows on $G$ induced by nilpotent or abelian elements of the associated Lie algebra are topologically conjugate.

Finally, in Section 4, we consider semidirect products $G=H V$, where $H$ is an arbitrary Lie group and $V$ is isomorphic to $R^{n}$. Here again we use the lifting result from Section 2 to show that the topological conjugacy class of a flow on $G$ induced by an element $(A, b)$ of the Lie algebra $g=h V$ does not depend on $b$.

\section{General case}

Considering several approaches to topological conjugacy, we emphasize the concept that we use in this paper. Let $M$ be a smooth manifold and $X(M)$ the space of smooth vector fields on $M$. Let $X_{1}, X_{2} \in X(M)$ be complete vector fields and $\Phi_{1}$ and $\Phi_{2}$ their associated flows (or dynamical systems). The flows $\Phi_{1}$ and $\Phi_{2}$, or the vector fields $X_{1}, X_{2}$, are called topologically conjugate if there exists a homeomorphism $h: M \rightarrow M$ such that $h\left(\Phi_{1}(t, x)\right)=\Phi_{2}(t, h(x))$ for all $x \in M$ and $t \in R$, and $h$ is called a topological conjugacy. If $h$ is a $C^{k}$-diffeomorphism, then $\Phi_{1}$ and $\Phi_{2}$ are said to be $C^{k}$-conjugate.

The flows considered here are associated with complete vector fields on a differentiable manifold, more precisely with left invariant vector fields on Lie groups. We analyze, in particular situations, combinations of flows that 
come from the exponential of elements in the Lie algebra associated with the Lie group.

An immediate case of conjugation between flows is taking similar matrices. In this case, the associated flows are conjugate by a linear (and hence differentiable) map, namely the similarity transformation of the corresponding matrices. More specifically, for two linear flows $\Phi$ and $\Psi$ in $R^{d}$, associated with $\dot{x}=A x$ and $\dot{x}=B x$, respectively, we have: $\Phi$ and $\Psi$ are $C^{k}$-conjugate for $k \geq 1$ if and only if $A$ and $B$ are similar, i.e., $A=T B T^{-1}$ for some $T \in \operatorname{Gl}(d, R)$. Each of these statements implies that $A$ and $B$ have the same eigenvalue structure and (up to a linear transformation) the same generalized real eigenspace structure. In particular, the $C^{k}$-conjugacy classes are exactly the real Jordan canonical form equivalence classes in $g l(d, R)$.

Due to this fact, the more interesting situation here is conjugation by a homeomorphism instead of a diffeomorphism. In this context, the existence of homeomorphic transversal sections (related to the flows) gives general results. One particular case is the known result: Given two hyperbolic matrices $A, B \in g l(d, R)$ whose stable subspaces have the same dimension it follows that the flows $\mathrm{e}^{A t} x$ and $\mathrm{e}^{B t} x$ are topologically conjugate. In the proof of this result, the existence of spheres, corresponding to adapted norms, such that the respective flows cross these spheres just once is essential.

Motivated by this we give the following definition:

Definition 1. Consider a topological space $M$ and a flow $\Phi: R \times M \longrightarrow$ $M$. A transversal section of $\Phi$ is a pair $(Z, \tau)$ where $Z$ is a subset of $M$ and $\tau: M \rightarrow R$ is a continuous map such that for all $x \in M$ it holds that $\Phi(t, x) \in Z$ if and only if $t=\tau(x)$.

Remark 2. Note that the existence of a transversal section is a very restrictive property for a flow. For example, assume that the flow $\Phi$ : $R \times M \rightarrow M$ has an equilibrium $x \in M$. Then obviously for any transversal section $(Z, \tau)$, the set $Z$ must contain $x$. But this implies $\Phi(t, x)=x \in Z$ for all $t \in R$, which contradicts the definition of a transversal section. Similarly, one sees that $\Phi$ cannot have any periodic trajectories. More generally, the existence of a transversal section implies that all $\alpha$ - and $\omega$-limit sets of the corresponding flow are empty. Indeed, assume that $\Phi\left(t_{n}, x\right)$ converges to $y \in M$ for some $x \in M$ and a sequence $t_{n} \rightarrow \infty$. Then

$$
\tau\left(\Phi\left(t_{n}, x\right)\right)=\tau(x)-t_{n} \rightarrow-\infty,
$$


which contradicts continuity of $\tau$ at $y$. So, in particular, flows on compact metrizable spaces do not admit transversal sections.

Now, the existence of homeomorphic transversal sections provides the following general result:

Theorem 3. Let $\Phi_{i}, i=1,2$, be two flows on a topological space $M$ with respective transversal sections $\left(Z_{i}, \tau_{i}\right), i=1,2$. Suppose that $Z_{1}$ is homeomorphic to $Z_{2}$. Then $\Phi_{1}$ and $\Phi_{2}$ are topologically conjugate.

Proof. Define $h: M \rightarrow M$ by $h(x):=\Phi_{2}\left(-\tau_{1}(x), h_{0}\left(\Phi_{1}\left(\tau_{1}(x), x\right)\right)\right)$, where $h_{0}: Z_{1} \rightarrow Z_{2}$ is a homeomorphism. We prove that $h$ is a topological conjugacy.

We begin proving that the conjugacy equation holds. First note that if $x \in M, t \in R$, and $s=\tau_{1}\left(\Phi_{1}(t, x)\right)$, then $\Phi_{1}\left(s, \Phi_{1}(t, x)\right)=\Phi_{1}(s+t, x) \in Z_{1}$. This implies $s+t=\tau_{1}(x)$, hence $s=\tau_{1}\left(\Phi_{1}(t, x)\right)=\tau_{1}(x)-t$.

Now we can prove that $h$ is conjugation. In fact,

$$
\begin{aligned}
h\left(\Phi_{1}(t, x)\right) & =\Phi_{2}\left(-\tau_{1}\left(\Phi_{1}(t, x)\right), h_{0}\left(\Phi_{1}\left(\tau_{1}\left(\Phi_{1}(t, x)\right), \Phi_{1}(t, x)\right)\right)\right) \\
& =\Phi_{2}\left(t-\tau_{1}(x), h_{0}\left(\Phi_{1}\left(\tau_{1}(x)-t, \Phi_{1}(t, x)\right)\right)\right) \\
& =\Phi_{2}\left(t-\tau_{1}(x), h_{0}\left(\Phi_{1}\left(\tau_{1}(x), x\right)\right)\right) \\
& =\Phi_{2}\left(t, \Phi_{2}\left(-\tau_{1}(x), h_{0}\left(\Phi_{1}\left(\tau_{1}(x), x\right)\right)\right)\right)=\Phi_{2}(t, h(x)) .
\end{aligned}
$$

To see that $h$ is injective, suppose that $h\left(x_{1}\right)=h\left(x_{2}\right)$. Take $\tau:=\tau_{1}\left(x_{1}\right)$. In this case

$$
h\left(\Phi_{1}\left(\tau, x_{1}\right)\right)=\Phi_{2}\left(\tau, h\left(x_{1}\right)\right)=\Phi_{2}\left(\tau, h\left(x_{2}\right)\right)=h\left(\Phi_{1}\left(\tau, x_{2}\right)\right) .
$$

Then

$$
h\left(\Phi_{1}\left(\tau, x_{1}\right)\right)=h\left(\Phi_{1}\left(\tau, x_{2}\right)\right) \in Z_{2} \text {, since } \Phi_{1}\left(\tau_{1}, x_{1}\right) \in Z_{1} \text { and } h\left(Z_{1}\right)=Z_{2} .
$$

Since $h$ maps only $Z_{1}$ to $Z_{2}$, it follows that $\Phi_{1}\left(\tau, x_{2}\right) \in Z_{1}$. Hence, by uniqueness, $\tau=\tau_{1}\left(x_{1}\right)=\tau_{1}\left(x_{2}\right)$. Now using that

$$
h_{0}\left(\Phi_{1}\left(\tau, x_{1}\right)\right)=h\left(\Phi_{1}\left(\tau, x_{1}\right)\right)=h\left(\Phi_{1}\left(\tau, x_{2}\right)\right)=h_{0}\left(\Phi_{1}\left(\tau, x_{2}\right)\right)
$$

and knowing that $h_{0}$ is injective, we have

$$
\Phi_{1}\left(\tau, x_{1}\right)=\Phi_{1}\left(\tau, x_{2}\right), \text { therefore } x_{1}=x_{2} .
$$


To show that $h$ is surjective, take $y \in M$. Then $\Phi_{2}\left(\tau_{2}(y), y\right) \in Z_{2}$. Hence, there exists $z \in Z_{1}$ such that $h_{0}(z)=\Phi_{2}\left(\tau_{2}(y), y\right)$. Let $x:=\Phi_{1}\left(-\tau_{2}(y), z\right)$. Using that $\tau_{1}(x)=\tau_{2}(y)$, it is not difficult prove that $h(x)=y$.

The map $h$ is continuous, since it is given by compositions of continuous maps. Finally, exchanging the roles of $\Phi_{1}$ and $\Phi_{2}$ we see that also $h^{-1}$ is continuous.

Let $M$ and $P$ be topological spaces and $\pi: M \times P \rightarrow M$ the canonical projection. If $\Phi$ is a flow on $M \times P$, which is semiconjugate to a flow $\widetilde{\Phi}$ on $M$ via the map $\pi$, then a transversal section of $\widetilde{\Phi}$ lifts to a transversal section of $\Phi$. This gives the following result:

Corollary 4. Let $M$ and $P$ be topological spaces and $\pi: M \times P \rightarrow M$ the canonical projection. Assume that $\widetilde{\Phi}_{1}$ and $\widetilde{\Phi}_{2}$ are flows on $M$, and $\Phi_{1}$ and $\Phi_{2}$ flows on $M \times P$ such that the diagram, given by $\Phi_{i}(t, \cdot): M \times P \rightarrow$ $M \times P, \pi: M \times P \rightarrow M$ and $\widetilde{\Phi}_{i}(t, \cdot): M \rightarrow M$, commutes for every $t \in R$. Suppose that there exist transversal sections $\left(\widetilde{W}_{i}, \widetilde{\tau}_{i}\right)$ for the flows $\widetilde{\Phi}_{i}$ such that $\widetilde{W}_{1}$ is homeomorphic to $\widetilde{W}_{2}$. Then $\Phi_{1}$ is topologically conjugate to $\Phi_{2}$.

Proof. Denote by $\widetilde{h}: \widetilde{W}_{1} \rightarrow \widetilde{W}_{2}$ the homeomorphism between the transversal sections and let $W_{i}:=\pi^{-1}\left(\widetilde{W}_{i}\right), i=1,2$. Then define

$$
h: W_{1} \rightarrow W_{2}, \quad h(x, y):=(\widetilde{h}(x), y) .
$$

It is clear that $h$ is a homeomorphism. Now, define $\tau_{i}: M \times P \rightarrow$ by $\tau_{i}:=\widetilde{\tau}_{i} \circ \pi, i=1,2$. Then

$$
\begin{aligned}
\Phi_{i}(t,(x, y)) \in W_{i} & \Leftrightarrow \pi\left(\Phi_{i}(t,(x, y))\right) \in \widetilde{W}_{i} \\
& \Leftrightarrow \widetilde{\Phi}_{i}(t, x) \in \widetilde{W}_{i} \\
& \Leftrightarrow t=\widetilde{\tau}_{i}(x)=\widetilde{\tau}_{i}(\pi(x, y))=\tau_{i}(x, y) .
\end{aligned}
$$

This proves that $\left(W_{i}, \tau_{i}\right)$ is a transversal section for the flow $\Phi_{i}$, which implies the result.

\section{Lie group case}

We begin this section giving necessary notation and background results about Lie groups and their associated Lie algebras.

Denote by $G$ a connected noncompact Lie group and let $g$ be its Lie algebra, that is, the tangent space of $G$ at the identity element $e \in G, T_{e} G$. Recall that if we consider the set $X^{e}(G)$ of left invariant vector fields on 
$G$ with the Lie bracket of vector fields, then $X^{e}(G)$ can be identified with $T_{e} G$.

Consider a right invariant vector field $X$ on $G$ and let $\Phi_{t}$ denote its flow, that is, the solution of the differential equation $\dot{g}=X(g)$. Then $X$ is a complete vector field, i.e., all solutions $\Phi_{t}(g)$ extend for all $t \in R$. With these trajectories we define the exponential map $\exp (t X)=\Phi_{t}(e)$ and obtain that exp : $g \rightarrow G$ is a diffeomorphism between a neighborhood of $0 \in g$ and a neighborhood of $e \in G$.

Now suppose that $G$ is a semisimple Lie group. As $g$ is semisimple, we have the Iwasawa decomposition, that is, it is known that $g$ is decomposed as $g=k \oplus a \oplus n^{+}$, where $k$ is the compact subspace associated to a Cartan involution, $a$ is the maximal abelian subalgebra in $s, n^{+}=\sum_{\alpha \in \Pi^{+}} g_{\alpha}$ and $g_{\alpha}$ stands for the $\alpha$-root space. Here, $\Pi$ is the set of roots of the pair $(g, a)$ and $\Pi^{+}$is the set of positive (respectively simple) roots. Now consider the connected subgroups $K, A$ and $N$ generated, respectively, by $\exp k$, $\exp a$ and $\exp n^{+}$. The map $(k, a, n) \in K \times A \times N \mapsto k a n \in K A N$ is a diffeomorphism between $K \times A \times N$ and $G$, and the decomposition $G=$ $K A N$ is called general Iwasawa decomposition.

\subsection{Nilpotent and abelian case}

In the case of a simply connected abelian Lie group any two flows are topologically conjugate. In fact, any isomorphism that relates two left invariant vector fields is a topological conjugacy between the corresponding flows. This idea can not be used in the case of a nilpotent Lie group. But using results of the previous section we show in this section that any two flows of nonzero left invariant vector fields on a simply connected nilpotent Lie group are topologically conjugate. We do this by constructing transversal sections for the corresponding flows.

Let $N$ be a simply connected nilpotent Lie group. It is known that $N$ is isomorphic to its Lie algebra $n$, via the exponential map, provided with the Campbell-Hausdorff product. The Campbell-Hausdorff series of $n$ is a finite sum. We can write the Campbell-Hausdorff product of $X, Y \in n$ as

$$
X * Y=X+Y+R_{2}+R_{3}+\cdots+R_{k},
$$

where $R_{i}$ is given by the brackets of $X, Y$ and $R_{i} \in n^{i}$, where $0=n^{k+1} \subset$ $n^{k} \subset \cdots \subset n^{2} \subset n$ is the descending central series of $n$.

Identifying $N$ with $(n, *)$, via the exponential map, the flow $\Phi(t, X)$ associated with $A \in n$ is the map $\Phi(t, X)=t A * X$, where $t \in R$ and $X \in n$. 
Proposition 1. In a simply connected nilpotent Lie group $N$, every flow induced by a nonzero element of the associated Lie algebra $n$ has a transversal section.

Proof. Take $A \in n$ with $A \neq 0$. Then there exists a transversal section in $n$ of $\Phi(t, X)=t A * X$, which is shown as follows: Let $j$ be a positive integer such that $A \in n^{j}$ but $A \notin n^{j+1}$. Consider a hyperplane $h$ of $n$ containing $n^{j+1}$ and such that $A \notin h$. If $X \in n$ and $t \in R$, knowing that $A \in n^{j}$ it follows that any bracket of $t A$ and $X$ is contained in $n^{j+1} \subset h$. Hence, $t A * X \in h$ if and only if $t A+X \in h$. As $h$ is a hyperplane not containing $A$, there exists an unique $t \in R$ such that $t A+X \in h$. Now define $\tau: n \rightarrow R$ by $\tau(X)=t$ if and only if $t A+X \in h$. It is clear that $\tau$ is continuous and therefore $(h, \tau)$ is a transversal section of the flow of $A$.

Theorem 2. Any two flows on a simply connected nilpotent Lie group $N$ induced by nonzero elements of the Lie algebra are topologically conjugate.

Proof. Take nonzero $A, B \in n$. Consider hyperplanes $h_{A}, h_{B} \subset n$ that are transversal to $A$ and $B$, respectively. As $h_{A}$ and $h_{B}$ are homeomorphic, the result follows from Theorem 3 .

The following corollary is immediate.

Corollary 3. Any two flows on a simply connected abelian Lie group induced by nonzero elements of the Lie algebra are topologically conjugate.

Example 4. On a nilpotent group which is not simply connected two flows are not necessarily topologically conjugate. Indeed, consider the 2dimensional torus $T^{2}=R^{2} / Z^{2}$ and an element $A=\left(a_{1}, a_{2}\right)$ of its Lie algebra ${ }^{2}$. The corresponding flow is

$$
\varphi\left(t, X+{ }^{2}\right)=t A+X+Z^{2} .
$$

If $A=(1,1)$, then obviously every point $X+Z^{2} \in T^{2}$ is 1-periodic. If $A=(1 / 2,1 / 2)$, then every point is 2 -periodic, and if $A=(1, \sqrt{2})$, no point is periodic. Since topological conjugacies map periodic orbits to periodic orbits preserving the period, this proves the assertion.

\subsection{Semi-simple case}

Let $G$ be a noncompact connected semisimple Lie group and $g$ its Lie algebra. In this section, we prove that the flows of two hyperbolic or nilpotent elements of $g$ are topologically conjugate in $G$. 
First we give some notation and background results about Lie groups and Lie algebras that will be used here.

Take the Iwasawa decomposition of $g, g=k \oplus a \oplus n^{+}$and the respective Iwasawa decomposition of $G, G=K A N$. It is not difficult to see that the order in which the groups appear in the Iwasawa decomposition can be changed. However, we will make a quick comment. First note that the map $g \mapsto g^{-1}$ is a diffeomorphism of $G$. Hence, $G=N A K$. Now consider a subgroup $D(p) \subset \operatorname{Gl}(p, R)$ of diagonal matrices and $N(p) \subset \mathrm{Gl}(p, R)$ the subgroup of upper triangular matrices. Take $n \in N(p)$ and $a \in D(p)$. Suppose that $n=\left(n_{i j}\right)$, where $n_{i i}=1$ and $n_{i j}=0$ if $i>j$ and suppose also that $a=\operatorname{diag}\left(a_{1}, a_{2}, \ldots, a_{p}\right)$. Then $n a=a n^{\prime}$, where $n^{\prime}=\left(n_{i j}^{\prime}\right)$ with $n_{i j}^{\prime}=\frac{n_{i j} a_{j}}{a_{i}}$. As $n^{\prime} \in N(p)$, then $N(p) D(p)=D(p) N(p)$. Now considering the adjoint group $\operatorname{Ad}(G)$ as a closed subgroup of $\operatorname{Gl}(p, R)$, the commutative property between $N(p)$ and $D(p)$ can be transferred to $\operatorname{Ad}(N)$ and $\operatorname{Ad}(A)$, and hence to $N$ and $A$. Therefore, $N A=A N$ and then we conclude that $G=K A N=N A K=A N K$.

Now, consider the canonical projection

$$
\pi: G=A N K \rightarrow A N K / N K
$$

and identify $A N K / N K$ with $A$.

Take $X_{1}, X_{2} \in a$ and the respective flows

$$
\Phi_{i}: R \times G \rightarrow G, i=1,2 \text {, given by } \Phi_{i}(t, g)=\exp \left(t X_{i}\right) g .
$$

The restrictions of these flows to $A$,

$$
\Phi_{i}^{A}: R \times A \rightarrow A, \quad(t, a) \mapsto \exp \left(t X_{i}\right) a, \quad i=1,2,
$$

make the diagram, given by $\Phi_{i}(t, \cdot): G \rightarrow G, \pi: G \rightarrow A$ and $\Phi_{i}^{A}(t, \cdot): A \rightarrow$ $A$ commute.

This follows from the identities

$$
\pi\left(\exp \left(t X_{i}\right) g\right)=\pi(\underbrace{\exp \left(t X_{i}\right)}_{\in A} g_{A} g_{N} g_{K})=\exp \left(t X_{i}\right) g_{A}=\exp \left(t X_{i}\right) \pi(g),
$$

where $g=g_{A} g_{N} g_{K}$ is the Iwasawa decomposition of $g \in G$.

As $A$ is abelian and simply connected, then by Proposition 1, there exist transversal sections $h_{1}, h_{2} \subset A$ for $X_{1}$ and $X_{2}$, respectively. Moreover, now consider $\pi: N A K \rightarrow N A K / A K$, identify $N A K / A K$ with $N$, and take $X_{1}, X_{2} \in n$. By similarity to the abelian case we can guarantee that 
there exist homeomorphic transversal sections $h_{1}, h_{2} \subset N$ for $X_{1}$ and $X_{2}$, respectively. Hence, taking the lifts of these transversal sections to $G$, it follows from Corollary 4 that the flows of $X_{1}$ and $X_{2}$ on $G$ are topologically conjugate:

Theorem 5. For any nonzero $X_{1}, X_{2}$ in $a$ or $n$ the correspondent flows are topologically conjugate in $G$.

Example 6. Now we present an example showing that topological conjugacy in a Lie group does not imply topological conjugacy in the respective Euclidean space: Take the following elements in $\operatorname{sl}(3, R)$ :

$$
X=\operatorname{diag}(1,0,-1) \text { and } Y=\operatorname{diag}(3,-1,-2) .
$$

We see in the above result that the corresponding flows of $X$ and $Y$ are conjugate in $S l(3, R)$, but the corresponding flows in $R^{3}$ are not conjugate. In fact, for $a=(0, y, 0) \in R^{3}$ we have that $\exp (t X) y$ is constant. Then, if $h: R^{3} \rightarrow R^{3}$ is a conjugation between the flows of $X$ and $Y, \exp (t Y) h(a)$ should be constant for all $a$ and for all $t$. But this is possible only if $h(a)=0$ for all $a$. Therefore, $h$ can not be conjugation.

\section{Affine group case}

First we need some notation and definitions. Let $V$ be an $n$-dimensional real vector space and $H$ a Lie group that acts on $V$. Take the group $G=H V$ given by the semidirect product of $H$ and $V$. Recall that the affine group operation is defined by $(g, v) \cdot(h, w)=(g h, v+g w)$ for all $(g, v),(h, w) \in G$. Let $\pi: G \rightarrow H$ be the canonical projection of the affine group onto the Lie group $H$. The action of $G$ on $V$, given by $(g, v) \cdot w=g w+v$, with $(g, v) \in G$ and $w \in V$, is called an affine action. The natural action of $\pi(G)=H$ on $V$ is called a linear action. Denote by $g=h V$ the Lie algebra of $G$, where $h$ is the Lie algebra of $H$. Note that for simplicity we use the same product symbol for the group and algebra. If $\Phi(t, g)=\exp _{G}(t X) g$ is a flow on $G$, where $X=(A, b) \in g=h V$ and $g \in G$, we denote by $\Phi^{H}$ the flow on $H$ given by $\Phi^{H}(t, h)=\exp _{H}(t A) h$ with $h \in H$.

Now we give an idea about the motivation of this section. The main results in [2] and [3] say that the topological conjugacy of affine systems depends on the topological conjugacy of the linear parts. More specifically, take two affine flows $\Psi$ and $\hat{\Psi}$ and denote by $\Phi$ and $\hat{\Phi}$ the respective linear parts. If $\Phi$ and $\hat{\Phi}$ are topologically conjugate, then $\Psi$ and $\hat{\Psi}$ are topologically conjugate. 
Another motivation is that controllability of affine systems depends on the controllability in the linear part. That is, let $G=B V$ be an affine group, where $B$ is a semisimple Lie group that acts transitively on $V-\{0\}$. Let $S \subset G$ be a connected semigroup with nonempty interior. Suppose that the linear action of $\pi(S)$ is transitive on $V-\{0\}$. Then the affine action of $S$ on $V$ is transitive. (see Rocio, Santana, and Verdi [8]). Motivated by this, we have the following result (Proposition 2).

Lemma 1. For $X=(A, b) \in h V$, the flow $\Phi(t, g)=\exp _{G}(t X) g$ on $G$ is given by

$$
\exp _{G}(t X) \cdot(h, v)=\left(\exp _{H}(t A) h, \exp _{H}(t A) v+\int_{0}^{t} \exp \left(s a d_{A}\right) b d s\right) .
$$

Proof. By Theorem 3.1 in [6], the exponential map $\exp _{G}$ for a semidirect product $G=H W$ of arbitrary Lie groups $H$ and $W$ is given by

$$
\exp _{G}(\eta, \omega)=\left(\exp _{H}(\eta), E_{W}^{D}(\omega)\right)
$$

where $E_{W}^{D}$ is defined as follows: Let $g(t)$ be the solution of

$$
g^{\prime}(t)=d L_{g(t)} \gamma(t)
$$

with $g(0)=e_{G}, \gamma(t)=\exp (t D) \gamma_{0}, \gamma: R \rightarrow g$. Define

$$
E_{W}^{D}: g \rightarrow G, \quad E_{G}^{D}\left(\gamma_{0}\right):=g(1) .
$$

where $\eta$ is identified with $(\eta, 0)$ and hence induces a derivation $D=a d_{\eta}$ on the Lie algebra of $W$.

Now, consider the case $W=V$ : Here the left translations are the affine maps $L_{g}: x \mapsto g+x$. Hence, the differential equation (4.1) reduces to

$$
g^{\prime}(t)=\gamma(t)=\exp (t D) \gamma
$$

We obtain the solution by integration:

$$
g(t)=\int_{0}^{t} \exp (t D) \gamma d t
$$

Using that $a d_{t \eta}=t a d_{\eta}$, we thus obtain

$$
\begin{aligned}
\exp _{G}(t \eta, t \omega) & =\left(\exp _{H}(t \eta), \int_{0}^{1} \exp \left(s t a d_{\eta}\right) t \omega\right) \\
& =\left(\exp _{H}(t \eta), \int_{0}^{t} \exp \left(s a d_{\eta}\right) \omega d s\right)
\end{aligned}
$$


Now, for $X=(A, b) \in h V$ we have the following flow on $G$ :

$$
\exp _{G}(t X) \cdot(h, v)=\left(\exp _{H}(t A) h, \exp _{H}(t A) v+\int_{0}^{t} \exp \left(s a d_{A}\right) b d s\right) .
$$

This proves the assertion.

Proposition 2. Consider the affine group $G=H V$. Let $\pi: G \rightarrow H$ be the canonical projection. Take flows $\Phi_{i}(t, g)=\exp \left(t X_{i}\right) g, i=1,2$, on $G$ and suppose that there exist homeomorphic transversal sections for the flows $\Phi_{i}^{H}$. Then $\Phi_{1}$ is topologically conjugate to $\Phi_{2}$.

Proof. Let $X_{i}=\left(A_{i}, b_{i}\right) \in h V$ for $i=1,2$. Using the preceding lemma, for every $t \in R$ we have

$$
\begin{aligned}
\pi\left(\Phi_{i}(t,(h, v))\right) & =\pi\left(\exp _{G}\left(t\left(A_{i}, b_{i}\right)\right)(h, v)\right)=\exp _{H}\left(t A_{i}\right) h \\
& =\Phi_{i}^{H}(t, h)=\Phi_{i}^{H}(t, \pi(h, v)) .
\end{aligned}
$$

Equivalently, the diagram, given by $\Phi_{i}(t, \cdot): G \rightarrow G, \pi: G \rightarrow H$ and $\Phi_{i}^{H}(t, \cdot): H \rightarrow H$ commutes. Hence by Corollary 4 , this implies the proposition.

Remark 3. As a consequence we obtain that in the case of a nilpotent or abelian group $H$ any two flows on $G=H V$ are topologically conjugate. In fact, using the same notations as above, take flows $\Phi_{i}(t, g)=\exp \left(t X_{i}\right) g$, $i=1,2$, on $G$. Then $\Phi_{i}^{H}(t, h)=\exp _{H}\left(t A_{i}\right) h$ with $h \in H$ are flows on $H$. Hence, by Subsection 3.2 there exist transversal sections for $\Phi_{i}^{H}, i=$ 1,2 . Therefore, by the last proposition it follows that $\Phi_{1}$ is topologically conjugate to $\Phi_{2}$, if $H$ is nilpotent or abelian. Now suppose that $H$ is semisimple and consider the Iwasawa decomposition $H=A N K=N A K$. Take $X_{i}=\left(A_{i}, b_{i}\right) \in h V$, but with $A_{i} \in a$ or $A_{i} \in n$, where $a$ and $n$ are the Lie algebras of $A$ and $N$, respectively. Then again by Subsection 3.2 we have that the flows on $H, \Phi_{1}^{H}(t, h)=\exp _{H}\left(t A_{1}\right) h$ and $\Phi_{2}^{H}(t, h)=\exp _{H}\left(t A_{2}\right) h$ are topologically conjugate. Hence, by the last proposition it follows that these flows on $G=H V$ are topologically conjugate. 


\section{References}

[1] V. Ayala, F. Colonius and W. Kliemann, On topological equivalence of linear flows with applications to bilinear control systems, J. Dynam. Control Systems 13, pp. 337-362, (2007).

[2] F. Colonius and A. J. Santana; Stability and Topological Conjugacy for Affine Differential Equations, Bol. Soc. Parana. Mat. 26, pp. 141-151, (2009).

[3] F. Colonius and A. J. Santana; Topological conjugacy for affine-linear flows and control systems, Communications on Pure and Applied Analysis 10, pp. 847-857, (2001).

[4] M. W. Hirsch, S. Smale and R. L. Devaney, Differential Equations, Dynamical Systems and an Introduction to Chaos, Elsevier, London, (2004).

[5] A. Katok and B. Hasselblatt; Introduction to the Modern Theory of Dynamical Systems, 1st Edition, Cambridge University Press, Cambridge, (1996).

[6] M. Moskowitz and R. Sacksteder; The Exponential Map and Differential Equations on Real Lie Groups, Journal of Lie Theory 13, pp. 291$306,(2003)$.

[7] C. Robinson, Dynamical Systems. Stability, Symbolic Dynamics, and Chaos, 2nd Edition, CRC Press, London, (1999).

[8] O. G. Rocio, A. J. Santana and M. A. Verdi, Semigroups of Affine Groups, Controllability of Affine Systems and Affine Bilinear Systems in $S l(2,)^{2}$, SIAM J. Control Optim. 48, pp. 1080-1088, (2009).

\section{Christoph Kawan}

Institut für Mathematik

Universität Augsburg

Augsburg, Germany,

e-mail :christoph.kawan@math.uni-augsburg.de 
188 Christoph Kawan, Osvaldo G. Rocío and Alexandre J. Santana

\title{
Osvaldo G. Rocio
}

Departamento de Matemática

Universidade Estadual de Maringá

Maringá

Brazil

e-mail : rocio@uem.br

and

\author{
Alexandre J. Santana \\ Departamento de Matemática, \\ Universidade Estadual de Maringá \\ Maringá, \\ Brazil, \\ e-mail : ajsantana@uem.br
}

